1957 y Geranio-Fagetum Vigo \& Gil 1983). La flora pteridofítica con la que convive está formada por Asplenium scolopendrium L., A. trichomanes L., Athyrium filix-femina (L.) Roth, Dryopteris affinis (Lowe) Fraser-Jenkins subsp. affinis, D. filix-mas (L.) Schott, Polystichum aculeatum (L.) Roth y P. setiferum
(Forsskål) Woynar. Añadir al A.P.I.B. la cuadrícula DG 3.

Aceptado para su publicación en Julio de 1993

Dirección de los autores. L. Sáez: C/ Pare Francesc Molina, 14. 07003 Palma de Mallorca. X. Viñas y L. Vilar: Laboratorio de Botánica. Estudio General de Girona. Plaza Hospital, 6. 07071 Girona.

\title{
39. APORTACIONES AL CONOCIMIENTO DE LA PTERIDOFLORA IBÉRI- CA Y BALEAR: CW DE LA PENÍNSULA IBÉRICA
}

\author{
Miguel Angel MARTÍN BALLESTEROS, Ma Montserrat MARTÍNEZ ORTEGA, \\ Ma José PÉREZ HORNERO, Enrique RICO HERNÁNDEZ \\ y Ximena GIRÁLDEZ FERNÁNDEZ.
}

Palabras clave. Pteridófitos, corología, España.

Key words. Pteridoflora, CW Spain.

Como resultado de las herborizaciones de los últimos años y de la revisión de herbarios llevada a cabo para el estudio general de los pteridófitos del CW hispano, se pueden añadir varios puntos al APIB (Atlas de la Pteridoflora Ibérica y Balear). Las referencias sin pliegos testigo de herbario, han sido identificadas «in situ», sin recolectar material, dado lo escaso de la población.

Isoetes setaceum Lam.

CÁCERES: Moraleja, río Corzo, PE9531, 30-V-91, Giráldez, Rico et al. Añadir el punto PE4 en el mapa del APIB.

Equisetum ramosissimum Desf.

SALAMANCA: Ciudad Rodrigo, QE0896, 620 m, 23-X-92, Martín Ballesteros, Pérez Hornero \& Rico. ZAMORA:Villanueva del Campo, "Santa Cruz”, UM0148, 730 m, 26-IX-92, Martín Ballesteros, Martínez Ortega \& Rico, SALA 56250. Castroverde de Campos, carretera a Valderas, Km 10 "El Cañal", UM0453, 750 m, 26-IX-92, Martín Ballesteros, Martínez Ortega \& Rico, SALA 56251. Añadir los puntos QE1, UM1 y UM2 en el mapa del APIB.

Ophioglossum vulgatum L.

ÁVILA: Entre Navacepeda y Navalperal de Tormes, UK0709, 1350 m, 3-V-92, Rico, SALA 56248. Añadir el punto UK2 en el mapa del APIB. No conocemos citas anteriores de Avila y debe añadirse esta provincia en la síntesis genérica de López González (Ophioglossum L. In : S. Castroviejo et al. (Eds.), Flora Ibérica I: 35-37. 1986) 
Ophioglossum lusitanicum L.

SALAMANCA: Saucelle, salto, PF8445, 200 m, 11-III-94, Martín Ballesteros, Rico \& T. Romero, SALA 56247. Añadir el punto PF4 en el mapa del APIB.

\section{Osmunda regalis $\mathrm{L}$.}

CÁCERES: Entre Alcorneo y Valencia de Alcántara, PD5256, 550 m, 17-V-92, Martín Ballesteros, Martínez Ortega, Pérez Hornero \& Rico, SALA 56083. Hoyos, arroyo de Santa Catalina, PE9448, 30-V-91, Giráldez, Rico et al. . Añadir los puntos PD3 y PE4 en el mapa del APIB.

\section{Polypodium interjectum Shivas}

ZAMORA: Castro de Alcañices, salto de Castro, QG3406, $700 \mathrm{~m}, 15$-IV-82, Navarro \& C.J. Valle, SALAF 2548. Añadir el punto QG2 en el mapa del APIB.

\section{Cheilanthes maderensis Lowe \\ CÁCERES: Almoharín, QD5342, 380-420 m,} 26-I-90, Giráldez \& Rico, SALA 55686. Añadir el punto QD2 en el mapa del APIB.

\section{Cheilanthes tinaei Tod.}

SALAMANCA: Alaraz, río Gamo, UL01, 7X-90, Rico. Añadir el punto QE2 en el mapa del APIB.

\section{Cosentinia vellea (Aiton) Tod.}

CÁCERES: Torrejón el Rubio, escaleras del castillo de Monfragüe, QE5213, 400 m, 23-IV-94, Pérez Hornero, SALA 56253. SALAMANCA: Saucelle, Puerto de la Molinera, PE9044, $300 \mathrm{~m}$, 30-I-94, Rico \& T. Romero, SALA 56252. Añadir el punto QE2 en el mapa del APIB por la localidad cacereña. La salmantina es la segunda cita para esa provincia.

\section{Marsilea strigosa Willd.}

CÁCERES: Brozas, arroyo de las Cañas, PD9471, 250 m, 17-V-92, Martín Ballesteros, Martínez Ortega, Pérez Hornero \& Rico, SALA 55996. Añadir el punto PD3 en el mapa del APIB.

\section{Pteridium aquilinum (L.) Kuhn}

SALAMANCA: Vega de Tirados, Baños de Ledesma, cerro del Jardín, TL5650, 760 m, 3-VIII92, Martín Ballesteros, SALA 56044. Añadir el punto TL1 en el mapa del APIB.

Asplenium billotii F.W. Schultz

CÁCERES: Puerto de la Cobacha, carretera de Cáceres a Badajoz, QD0740, 380 m, 5-VI-90, Casaseca \& Rico, SALA 55884. Almoharín, QD5342, 380-420 m, 26-I-90, Giráldez \& Rico, SALA 55880. SALAMANCA: Cerralbo, río Huebra, Molino de Picones, QF0540, 600 m, 10-III-90, Giráldez \& Rico. Añadir los puntos QD2 y QF2 en el mapa del APIB.

\section{Asplenium onopteris $\mathrm{L}$.}

SALAMANCA: De Membribe a Navagallego, Peña Sta.Cruz, TL6310, 26-III-91, Giráldez \& Martín Ballesteros, SALA 51159. Añadir el punto TL2 en el mapa del APIB.

Asplenium septentrionale L.Hoffm.

SALAMANCA: San Miguel de Valero, arroyo de San Juan, TK5193, 820 m, 14-XI-93, Pérez Hornero, SALA 56254. Se trata de la segunda cita para la provincia de Salamanca y la más occidental conocida del Sistema Central.

\section{Ceterach officinarum Willd.}

CÁCERES: Hoyos, PE9449, 520 m, 23-X-92, Martín Ballesteros, Pérez Hornero \& Rico. ZAMORA: Salto de Castro, QG3407, 27-XII-90, Rico. Añadir los puntos QG2 y PE4 en el mapa del APIB.

\section{Cystopteris dickieana R. Sim.}

SALAMANCA: Las Veguillas, Peña Gudina, TL61, 13-VI-85, T. Monzón, SALA 46719. Añadir el punto TL2 en el mapa del APIB.

\section{Athyrium filix-femina (L.) Roth}

CÁCERES: Serradilla, QE41, 10-VI-81, Ladero \& C.J. Valle, SALAF 20607. Entre Alcorneo y Valencia de Alcántara, PD5256, 575 m, 17-V-92, Martín Ballesteros, Martínez Ortega, Pérez Hornero \& Rico, SALA 56221. Añadir los puntos QE2 y PD3 en el mapa del APIB.

\section{Cystopteris viridula (Desv.) Desv.}

CÁCERES: Subida a Casas del Castañar, TK5044, 500 m, 2-VII-92 Martín Ballesteros, Martínez Ortega, \& Pérez Hornero, SALA 56035. Añadir el punto TK2 en el mapa del APIB. 
Dryopteris affinis subsp. borreri (Newman) Fraser-Jenkins

CÁCERES: Serradilla, arroyo Barbaón, QE41, 16-VI-81, Ladero \& C. J. Valle, SALA 23354. ZAMORA: Villarino de Manzanas, arroyo Cabrón, QG0839, 740 m, 9-VII-91, Giráldez, Martínez Ortega, Pérez Hornero \& Rico, SALA 56233. Añadir los puntos QE2 y QG2 en el mapa del APIB.

Blechnum spicant (L.) Roth

CACERES: Entre Alcorneo y Valencia de Alcántara, PD5256, 575 m, 17-V-92 Martín Balles- teros, Martínez Ortega, Pérez Hornero \& Rico, SALA 56016. Añadir el punto PD3 en el mapa del APIB.

Aceptado para su publicación en Junio de 1994

Dirección de los autores. Departamento de Biología Vegetal (Botánica). Facultad de Biología. Universidad de Salamanca. Campus Miguel de Unamuno. 37007 Salamanca. 